\title{
CARLESON MEASURE CHARACTERIZATIONS OF BMOA ON PSEUDOCONVEX DOMAINS
}

\author{
Hyeonbae Kang and Hyungwoon Koo
}

\begin{abstract}
We give various versions of Carleson measure characterization of BMOA on pseudo-convex domains of finite type in $\mathbb{C}^{2}$ as well as on strongly pseudo-convex domains in $\mathbb{C}^{n}$.
\end{abstract}

\section{Introduction.}

The purpose of this paper is to prove the following complex version of a well known theorem of Fefferman and Stein $[\mathbf{F S}]$.

Theorem 1.1. Let $\Omega=\left\{z \in \mathbb{C}^{2}: r(z)>0\right\}$ be a bounded pseudo-convex domain of finite type in $\mathbb{C}^{2}$ where $r$ is a smooth real valued function such that $|\nabla r|=1$ on $\partial \Omega$. Then, for a holomorphic function $f$ in $\Omega$, the following are equivalent:

(1) $f \in B M O A$.

(2) $\quad d \mu(z)=r(z)^{-1}\left(\left|r(z) \nabla_{N} f(z)\right|^{2}+\left|\tau(\pi(z), r(z)) \nabla_{T} f(z)\right|^{2}\right) d m(z)$ is a Carleson measure, where $d m$ is the Lebesgue measure.

(3) $\quad d \mu(z)=r(z)^{-1}\left|\tau(\pi(z), r(z)) \nabla_{T} f(z)\right|^{2} d m(z)$ is a Carleson measure.

(4) $d \mu(z)=r(z)^{-1}\left|r(z) \nabla_{N} f(z)\right|^{2} d m(z)$ is a Carleson measure.

Here, BMOA and the Carleson measure are defined in terms of NagelStein-Wainger ball [NSW2] and $\tau(\zeta, \delta)$ is essentially the radius in complex tangential direction of the ball $B(\zeta, \delta)$. (For precise definitions, see Section 2.) We also have a similar theorem for strongly pseudo-convex domains in $\mathbb{C}^{n}$.

On the unit ball in $\mathbb{C}^{n}$, the equivalence of (1) and (2) was proved by Coifman, Rochberg, and Weiss using the Bergman metric [CRW]. The equivalence of (1) and (3) was proved by Choa and Choe using the PaleyLittlewood identity [CC]. Recently, M. Jevtic proved the equivalence of (1) and (4) $[\mathbf{J}]$. Notice that on the unit ball in $\mathbb{C}^{n}, \tau(\zeta, \delta) \approx \delta^{1 / 2}$ for any $\zeta$ and hence $\left(r\left|\nabla_{N} f\right|^{2}+\tau^{2} r^{-1}\left|\nabla_{T} f\right|^{2}\right) d m \approx r|\tilde{\nabla} f| d m$ where $\tilde{\nabla}$ is the gradient with respect to the Bergman metric. The Bergman metric on the unit ball is explicit. Moreover, the unit ball is homogeneous. These properties of the unit ball are essential ingredients in above mentioned works. However, these 
properties are absent on pseudo-convex domains. Quite recently, Krantz and Li showed that $f \in B M O A$ if and only if $r|\nabla f|^{2} d m$ is a Carleson measure and established the duality of $H^{1}$ and $B M O A$ on strongly pseudo-convex domains and weakly pseudo-convex domains of finite type in $\mathbb{C}^{2}[\mathbf{K L}]$.

The result of this paper refines the result in $[\mathbf{K L}]$ in tangential and normal directions in a precise way. The quantities $\left|r \nabla_{N} f\right|^{2}$ and $\left|\tau \nabla_{T} f\right|^{2}$ in Theorem 1.1 arise naturally since the maximum size polydisc contained in $\Omega$ has the radius proportional to $r$ in the normal direction and $\tau$ in the tangential direction.

To prove Theorem 1.1, we will use the result of Krantz-Li and some ideas in the proof of Fefferman-Stein theorem as appeared in $[\mathbf{S}]$, such as area integrals. Other ingredient for the proof in this paper is the derivative estimates of holomorphic functions on pseudo-convex domains obtained by Grellier [G].

This paper is organized as follows: In Section 2 we review some properties of nonisotropic balls defined in [NSW1, NSW2]. In Section 3 we derive some estimates of derivatives of holomorphic functions. In Section 4 we prove Theorem 1.1.

We use the notation $\lesssim: A \lesssim B$ means that $A \leq C B$ for some constant $C$ which does not depend on quantities to be estimated.

We wish to thank the anonymous referee for valuable suggestions.

\section{Nonisotropic Balls and Polydiscs.}

This section consists mostly of the facts from the well developed theory on the geometry of pseudo-convex domains of finite type [BDN, C, NSW1], [NSW2]. We need to review them for proper exposition of the result and proof in this paper.

Let $\Omega=\left\{z \in \mathbb{C}^{2}: r(z)>0\right\}$ be a smoothly bounded pseudo-convex domain in $\mathbb{C}^{2}$ where $r$ is a smooth real valued function with $|\nabla r|=1$ on $\partial \Omega$. Given a point $p \in \partial \Omega$, we may assume that $\frac{\partial r}{\partial x_{1}}(p)=1$ and $\frac{\partial r}{\partial y_{1}}(p)=$ $\frac{\partial r}{\partial x_{2}}(p)=\frac{\partial r}{\partial y_{2}}(p)=0$ where $z_{j}=x_{j}+y_{j}, j=1,2$. Near $p$, define vector fields $T$ and $L$ by

$$
T=\frac{\partial r}{\partial x_{1}} \frac{\partial}{\partial y_{1}}-\frac{\partial r}{\partial y_{1}} \frac{\partial}{\partial x_{1}} \quad \text { and } \quad L=\frac{\partial r}{\partial z_{1}} \frac{\partial}{\partial z_{2}}-\frac{\partial r}{\partial z_{2}} \frac{\partial}{\partial z_{1}} .
$$

Then $\Re L$, $\Im L$, and $T$ form a basis of the tangent space $T_{\zeta}(\partial \Omega)$ for $\zeta \in \partial \Omega$ near $p$.

For each $\zeta \in \partial \Omega$ near $p$, define $\lambda_{i_{1}, \ldots, i_{j}}(\zeta)$ by

$$
\left[L_{i_{j}},\left[\ldots\left[L_{i_{2}}, L_{i_{1}}\right] \ldots\right]\right](\zeta)=\lambda_{i_{1}, \ldots, i_{j}}(\zeta) T(\zeta) \quad \bmod \langle L, \bar{L}\rangle
$$


where [ , ] denotes the Lie bracket and $L_{i_{k}} \in\{L, \bar{L}\}$. Let

$$
\Lambda_{j}(p)^{2}=\sum_{k=2}^{j}\left|\lambda_{i_{1}, \ldots, i_{k}}(p)\right|^{2} .
$$

We say $p$ is of type $k$ if $\Lambda_{k}(p) \neq 0$ and $\Lambda_{j}(p)=0$ for $j=2, \ldots, k-1$. Suppose that every point on $\partial \Omega$ is of finite type and let $m$ be the maximum type of $\partial \Omega$. Let

$$
\Lambda(p, \delta)=\sum_{j=2}^{m} \Lambda_{j}(p) \delta^{j}
$$

and define $\tau(p, \delta)$ by

$$
\Lambda(p, \tau(p, \delta))=\delta
$$

One can easily see that

$$
\delta^{1 / 2} \lesssim \tau(p, \delta) \lesssim \delta^{1 / m}
$$

Following [NSW2], we now define a family of balls on $\partial \Omega$ which makes $\partial \Omega$ a space of homogeneous type in the sense of $[\mathbf{C W}]$. For $\zeta \in \partial \Omega$ near $p$ and small $\delta \geq 0$, say $0 \leq \delta \leq \delta_{0}$, let

$$
B(\zeta, \delta)=\exp _{\zeta}\{\alpha T+\beta L+\bar{\beta} \bar{L}:|\alpha|<\delta,|\beta|<\tau(\zeta, \delta)\}
$$

where $\exp _{\zeta}$ refers to the usual exponential map. Note that these balls are essentially twisted ellipsoids of radius $\tau(\zeta, \delta)$ in the complex tangential directions, and radius $\delta$ in the direction $T$. Let $d(\zeta, \xi)$ be the pseudo-distance associated with this family of balls, namely, $d(\zeta, \xi)=\inf \{\delta: \xi \in B(\zeta, \delta)\}$.

On the other hand, at every $p \in \partial \Omega$ there is a local coordinate system such that with this coordinate system the defining function $r$ becomes

$$
r\left(z_{1}, z_{2}\right)=x_{1}-h_{p}\left(y_{1}, z_{2}\right)
$$

with $h_{p}(0,0)=0, \nabla h_{p}(0,0)=0$, and $\frac{\partial^{k} h_{p}}{\partial z_{2}^{k}}(0,0)=\frac{\partial^{k} h_{p}}{\partial \bar{z}_{2}^{k}}(0,0)=0$ for $0 \leq k \leq$ $m$. Moreover, for all $\delta$ with $0<\delta<\delta_{0}$ (by shrinking $\delta_{0}$ if necessary),

$$
\Lambda\left(p, C_{1} \delta\right) \leq \sum_{i+j \leq m}\left|\frac{\partial^{i+j} h_{p}}{\partial z_{2}^{i} \partial \bar{z}_{2}^{j}}(0,0)\right| \delta^{i+j} \leq \Lambda\left(p, C_{2} \delta\right)
$$

where $C_{1}$ and $C_{2}$ are independent of $p$ and $\delta$. (See [BDN] or $[\mathbf{C}]$.) Then, one can easily see that, with the local coordinates,

$$
L=\left(1+g_{p}\left(z_{2}\right)\right)\left(\frac{\partial r}{\partial z_{1}} \frac{\partial}{\partial z_{2}}-\frac{\partial r}{\partial z_{2}} \frac{\partial}{\partial z_{1}}\right)
$$


where $g_{p}\left(z_{2}\right)$ is a function smooth in $p$ and $z_{2}$ and small uniformly in $p$ and $z_{2}$.

Lemma 2.1. There exists $C>0$ such that in terms of the local coordinates near $p \in \partial \Omega$,

$$
\left|z_{2} \frac{\partial r}{\partial z_{2}}(z)\right| \leq \Lambda\left(p, C\left|z_{2}\right|\right)
$$

Proof. By (2.6), we have

$$
z_{2} \frac{\partial r}{\partial z_{2}}(z)=-\sum_{\substack{i, j \geq 1 \\ i+j \leq m}} \frac{\partial^{i+j} h_{p}}{\partial z_{2}^{i} \partial \bar{z}_{2}^{j}}(0) z_{2}^{i}{\overline{z_{2}}}^{j}+O\left(\left|z_{1}\right|^{2}+\left|z_{1}\right|\left|z_{2}\right|+\left|z_{2}\right|^{m+1}\right) .
$$

Thus Lemma 2.1 follows from (2.7).

We now define a polydisc centered at $z \in \Omega$ near $p \in \partial \Omega$. Using the local coordinates near $p$, define

$$
P_{\epsilon}(z)=\left\{\xi=z+\zeta+\beta L(\pi(z)) \in \mathbb{C}^{2}:|\zeta|<\epsilon r(z),|\beta|<\epsilon \tau(p, r(z))\right\}
$$

where $\pi(z)$ is the normal projection of $z$ to $\partial \Omega$. Then $P_{\epsilon}(z)$ is of size $\epsilon r(z)$ in the complex normal direction and $\epsilon \tau(p, r(z))$ in the complex tangential direction. (See [NSW1].)

On $\Omega$ there are approach regions naturally associated with the nonisotropic distance: For $\zeta \in \partial \Omega$ and $\delta>0$, define the admissible approach region by

$$
\mathcal{A}_{\alpha}(\zeta)=\left\{w \in \Omega: d(\zeta, \pi(w))<\alpha r(w), r(w)<\delta_{0}\right\}
$$

The following lemma is well-known. (A complete proof can be found in $[\mathbf{K}]$.)

Lemma 2.2 [NSW1]. For each $\alpha>0$ and small $\epsilon>0$, there exists $\beta$ such that for all $p \in \partial \Omega$ and for all $z \in \mathcal{A}_{\alpha}(p)$

$$
P_{\epsilon}(z) \subset \mathcal{A}_{\beta}(p) \text {. }
$$

We now recall the definitions of BMOA and the Carleson measure on $\Omega$. Define a "tent" over a ball $B(\zeta, \delta) \subset \partial \Omega$ by

$$
B^{\#}(\zeta, \delta)=\{z \in \Omega: \pi(z) \in B(\zeta, \delta), r(z)<\delta\} .
$$

Notice that that $B(\zeta, \delta)$ is defined for all $\zeta \in \partial \Omega$ if $\delta \leq \delta_{0}$ and $\pi(z)$ is well-defined if $r(z) \leq \delta_{0}$ by shrinking $\delta_{0}$ if necessary. Let $\sigma$ be the surface 
area measure on $\partial \Omega$. A positive finite Borel measure $\mu$ is called a Carleson measure on $\Omega$ if there is $C>0$ such that for all $\zeta \in \partial \Omega$ and $0<\delta \leq \delta_{0}$,

$$
\mu\left(B^{\#}(\zeta, \delta)\right) \leq C \sigma(B(\zeta, \delta)) .
$$

A function $f \in H^{2}(\Omega)$ is called a function of bounded mean oscillation (BMOA) if

$$
\|f\|_{*}^{2}:=\sup _{\zeta \in \partial \Omega, 0<\delta \leq \delta_{0}} \frac{1}{\sigma(B(\zeta, \delta))} \int_{B(\zeta, \delta)}\left|f(\xi)-f_{B(\zeta, \delta)}\right|^{2} d \sigma(\xi)<\infty
$$

where $f_{B(\zeta, \delta)}=\sigma(B(\zeta, \delta))^{-1} \int_{B(\zeta, \delta)} f(\xi) d \sigma(\xi)$. (We use the same letter $f$ for the boundary value of $f$.)

Notice that for the definition of BMOA, we used the $L^{2}$ integral instead of $L^{1}$ integral as in the usual definition of BMO. This does not make any difference because of the John-Nirenberg inequality.

Before finishing this section, let us fix some notations for this paper. For $z \in \Omega$ with $r(z) \leq \delta_{0}$, let

$$
\tau(z)=\tau(\pi(z), r(z))
$$

Let $\nabla_{T}$ and $\nabla_{N}$ be the usual tangential and normal part of the Euclidean gradient. To keep notations short, let

$$
D_{T} f(z)=\tau(z) \nabla_{T} f(z) \text { and }|D f(z)|=\left|r(z) \nabla_{N} f(z)\right|+\left|D_{T} f(z)\right| .
$$

\section{Derivative Estimates.}

Grellier proved the following:

Lemma $3.1[\mathrm{G}$, Theorem A and B]. Let $z \in \Omega$ be a point near $\partial \Omega$. Then, for any $f$ holomorphic in $\Omega$ and a positive integer $k$,

$$
\begin{aligned}
|D f(z)| & \lesssim \frac{1}{\left|P_{\epsilon}(z)\right|} \int_{P_{\epsilon}(z)}|f(w)| d m(w), \\
\left|\tau^{k} \nabla_{T}^{k} f(z)\right| & \lesssim \frac{1}{\left|P_{\epsilon}(z)\right|} \int_{P_{\epsilon}(z)}|f(w)| d m(w) .
\end{aligned}
$$

Moreover, there exists a constant $C>0$ such that for any $t>0$ there exist $C(t)$ and $\delta_{0}$ such that for all $z$ with $|r(z)|<\delta_{0}$, we have

$$
|D f(z)| \leq \frac{C}{\left|P_{\epsilon}(z)\right|} \int_{P_{\epsilon}(z)}\left(C(t)\left|D_{T} f\right|+t \sum_{1 \leq i+j \leq(m-1)}\left|\tau^{i} r^{j} \nabla_{T}^{i} \nabla^{j} f\right|\right) d m .
$$


As consequences, we have the following:

Lemma 3.2. Let $k$ be a positive integer. Then for all $t>0$ there exists $\delta_{0}$ such that if $|r(z)|<\delta_{0}$,

$$
\begin{aligned}
\left|\tau^{k} \nabla_{T}^{k} f(z)\right| & \lesssim \frac{1}{\left|P_{\epsilon}(z)\right|} \int_{P_{\epsilon}(z)}|D f(w)| d m(w), \\
|D f(z)| & \lesssim \frac{1}{\left|P_{\epsilon}(z)\right|} \int_{P_{\epsilon}(z)}\left(\left|D_{T} f(w)\right|+t|r(w) \nabla f(w)|\right) d m(w) .
\end{aligned}
$$

Proof. If we use the local coordinate, then by $(2.8), L^{k}$ is a linear combination of differential operators of the form

$$
D_{\alpha, \beta}=\left(\prod_{j=1}^{k} \frac{\partial^{\alpha_{j}+\beta_{j}} r}{\partial z_{1}^{\alpha_{j}} \partial z_{2}^{\beta_{j}}}\right) \frac{\partial^{\alpha+\beta}}{\partial z_{1}^{\alpha} \partial z_{2}^{\beta}}
$$

where $\alpha_{j}+\beta_{j}, \alpha+\beta \geq 1$ and $\alpha+\sum \alpha_{j}, \beta+\sum \beta_{j} \leq k$. By the Taylor expansion of $r$ and (2.7) we have

$$
\frac{\partial^{\alpha_{j}+\beta_{j}} r}{\partial z_{1}^{\alpha_{j}} \partial z_{2}^{\beta_{j}}}(z)=O\left(r^{1-\alpha_{j}} \tau^{-\beta_{j}}\right) .
$$

Thus

$$
D_{\alpha, \beta}=O\left(r^{k-\sum \alpha_{j}} \tau^{-\sum \beta_{j}}\right) \frac{\partial^{\alpha+\beta}}{\partial z_{1}^{\alpha} \partial z_{2}^{\beta}}=O\left(r^{\alpha} \tau^{\beta-k}\right) \frac{\partial^{\alpha+\beta}}{\partial z_{1}^{\alpha} \partial z_{2}^{\beta}} .
$$

Notice that $r(w) \approx r(z)$ and $\tau(p, r(w)) \approx \tau(p, r(z))$ if $w \in P_{\epsilon}(z)$. Therefore, using the Cauchy estimates for the derivatives of $f$, we have

$$
\left|\tau^{k} D_{\alpha, \beta} f(z)\right| \lesssim \frac{1}{\left|P_{\epsilon}(z)\right|} \int_{P_{\epsilon}(z)}\left(\left|r(w) \frac{\partial f}{\partial z_{1}}(w)\right|+\left|\tau(p, r(w)) \frac{\partial f}{\partial z_{2}}(w)\right|\right) d m(w) .
$$

By (2.8) and Lemma 2.1, for $w \in P_{\epsilon}(z)$

$$
L f(w)=O(1) \frac{\partial f}{\partial z_{2}}(w)+O\left(\frac{\Lambda\left(p,\left|w_{2}\right|\right)}{\left|w_{2}\right|}\right) \frac{\partial f}{\partial z_{1}}(w) .
$$

Since $\frac{\Lambda\left(p,\left|w_{2}\right|\right)}{\left|w_{2}\right|}$ is an increasing function of $\left|w_{2}\right|$ and $\left|w_{2}\right| \lesssim \tau(p,|w|)$ for $w \in$ $P_{\epsilon}(z)$, it follows that

$$
\left|\tau(p, r(w)) \frac{\partial f}{\partial z_{2}}(w)\right| \lesssim|\tau(p, r(w)) L f(w)|+\left|r(w) \frac{\partial f}{\partial z_{1}}(w)\right|
$$

Thus we have (3.4).

Notice that by (3.1), (3.2), and (3.4) we have for $i+j \geq 1$,

$$
\left|\tau^{i} r^{j} \nabla_{T}^{i} \nabla^{j} f(z)\right| \lesssim \frac{1}{\left|P_{\epsilon}(z)\right|} \int_{P_{\epsilon}(z)}|D f(w)| d m(w) .
$$

Thus by (3.3) we have (3.5). This completes the proof. 


\section{Proof of Theorem 1.1.}

In this section, we prove the main theorem of this paper, Theorem 1.1. Since $(2) \Rightarrow(3)$ is trivial, we will prove $(1) \Rightarrow(2),(3) \Rightarrow(4)$, and $(4) \Rightarrow(1)$.

$(1) \Rightarrow(2)$. Suppose $f \in$ BMOA. It is known that $r|\nabla f|^{2} d m$ is a Carleson measure (see $[\mathbf{K L}]$ ). Thus it suffices to show that $\frac{\tau^{2}}{r}\left|\nabla_{T} f\right|^{2} d m$ is a Carleson measure. Fix a ball $B=B\left(\zeta_{0}, \delta\right) \subset \partial \Omega$ and set $\zeta^{\delta}=\zeta-\delta \nu_{\zeta}$ where $\nu_{\zeta}$ is the unit outward normal vector at $\zeta \in \partial \Omega$. Using Hardy's inequality we have

$$
\begin{aligned}
\int_{B^{\#}} \frac{\tau^{2}}{r}\left|\nabla_{T} f\right|^{2} d m & =\int_{B} \int_{0}^{\delta} \frac{\tau^{2}}{r}\left|\nabla_{T} f\right|^{2} d r d \sigma \\
& \leq \int_{B} \int_{0}^{\delta} \frac{\tau^{2}}{r}\left(\int_{r}^{\delta}\left|\nabla \nabla_{T} f\right| d t+\left|\nabla_{T} f\left(\zeta^{\delta}\right)\right|\right)^{2} d r d \sigma \\
& \lesssim \int_{B} \int_{0}^{\delta} r \tau^{2}\left|\nabla \nabla_{T} f\right|^{2} d r d \sigma+\int_{B} \tau(\zeta, \delta)^{2}\left|\nabla_{T} f\left(\zeta^{\delta}\right)\right|^{2} d \sigma(\zeta) \\
& \lesssim \int_{B^{\#}} r \tau^{2}\left|\nabla \nabla_{T} f\right|^{2} d m+\int_{B} \tau(\zeta, \delta)^{2}\left|\nabla_{T} f\left(\zeta^{\delta}\right)\right|^{2} d \sigma(\zeta) .
\end{aligned}
$$

By Lemma 3.1 we have

$$
\begin{aligned}
\int_{B^{\#}} r \tau^{2}\left|\nabla \nabla_{T} f\right|^{2} d m & \lesssim \int_{B^{\#}} r \tau^{2}\left(\left|\nabla_{T} \nabla f\right|+|\nabla f|\right)^{2} d m \\
& \lesssim \int_{(2 B)^{\#}} r|\nabla f|^{2} d m \\
& \leq C \sigma(B)
\end{aligned}
$$

since $r|\nabla f|^{2}$ is a Carleson measure.

Note that for $z \in \Omega$

$$
\begin{aligned}
\frac{\partial f}{\partial z_{j}}(z) & =\int_{\Omega} K(z, \zeta) \frac{\partial}{\partial \zeta_{j}}\left(f-f_{B}\right)(\zeta) d m(\zeta) \\
& =\int_{\Omega} \frac{\partial}{\partial \zeta_{j}}\left[K(z, \zeta)\left(f(\zeta)-f_{B}\right)\right] d m(\zeta)
\end{aligned}
$$

where $K(\cdot, \cdot)$ is the Bergman kernel on $\Omega$. Thus by divergence theorem we have

$$
\frac{\partial f}{\partial z_{j}}(z)=\int_{\partial \Omega} K(z, \zeta)\left[f(\zeta)-f_{B}\right] \frac{\partial r}{\partial \zeta_{j}}(\zeta) d m(\zeta) .
$$

We now use the local coordinate system near $\zeta_{0}$ as decribed in Section 2 . Since $\left|\frac{\partial r}{\partial z_{1}}\right| \leq C$,

$$
\left|\nabla_{T} f\left(\zeta^{\delta}\right)\right| \leq\left|\frac{\partial r}{\partial z_{1}}\left(\zeta^{\delta}\right) \frac{\partial f}{\partial z_{2}}\left(\zeta^{\delta}\right)\right|+\left|\frac{\partial r}{\partial z_{2}}\left(\zeta^{\delta}\right) \frac{\partial f}{\partial z_{1}}\left(\zeta^{\delta}\right)\right|
$$




$$
\begin{aligned}
& \lesssim \int_{\partial \Omega}\left|K\left(\zeta^{\delta}, \eta\right)\left(f(\eta)-f_{B}\right)\right|\left|\frac{\partial r}{\partial \eta_{2}}(\eta)\right| d \sigma(\eta) \\
& \quad+\left|\frac{\partial r}{\partial z_{2}}\left(\zeta^{\delta}\right)\right| \int_{\partial \Omega}\left|K\left(\zeta^{\delta}, \eta\right)\left(f(\eta)-f_{B}\right)\right| d \sigma(\eta) \\
& :=I+I I .
\end{aligned}
$$

The following size estimation of the Bergman kernel is well-known (see [NRSW] or [Mc]): For $z \in \Omega$ and $\zeta \in \partial \Omega$,

$$
|K(z, \zeta)| \lesssim \frac{1}{d(\zeta, \pi(z)) \sigma(B(\zeta, d(\zeta, \pi(z))))}
$$

Thus by Lemma 2.1 we have

$$
\begin{aligned}
I \lesssim \int_{B} & \frac{1}{\sigma(B) \delta}\left|f(\eta)-f_{B}\right| \frac{\delta}{\tau(\zeta, \delta)} d \sigma(\eta) \\
& +\sum_{j=1}^{\infty} \int_{2^{j} B \backslash 2^{j-1} B} \frac{1}{\sigma\left(2^{j} B\right) 2^{j} \delta}\left|f(\eta)-f_{B}\right| \frac{2^{j} \delta}{\tau\left(\zeta, 2^{j} \delta\right)} d \sigma(\eta) .
\end{aligned}
$$

If $f \in B M O A$, then

$$
\int_{2^{j} B}\left|f(\eta)-f_{B}\right| d \sigma(\eta) \lesssim j \sigma\left(2^{j} B\right)\|f\|_{*}
$$

(see $[\mathbf{S 2}])$ and hence

$$
I \lesssim \sum_{j=0}^{\infty} \frac{j+1}{\tau\left(\zeta, 2^{j} \delta\right)}\|f\|_{*} \lesssim \frac{\|f\|_{*}}{\tau(\zeta, \delta)}
$$

In the exactly same way, we have

$$
I I \lesssim \frac{\|f\|_{*}}{\tau(\zeta, \delta)}
$$

Thus we have

$$
\left|\nabla_{T} f\left(\zeta^{\delta}\right)\right| \lesssim \frac{\|f\|_{*}}{\tau(\zeta, \delta)}
$$

Therefore

$$
\int_{B} \tau(\zeta, \delta)^{2}\left|\nabla_{T} f\left(\zeta^{\delta}\right)\right|^{2} d \sigma(\zeta) \leq C \sigma(B)\|f\|_{*}^{2} .
$$

This completes the proof of $(1) \Rightarrow(2)$. 
$(3) \Rightarrow(4)$. Suppose that $r^{-1}\left|D_{T} f\right|^{2} d m$ is a Carleson measure and let $B(\zeta, \delta) \subset \partial \Omega$. Let $M$ be the doubling constant on $\partial \Omega$, namely, for all $\zeta \in \partial \Omega$ and $\delta>0$,

$$
\sigma(B(\zeta, 2 \delta)) \leq M \sigma(B(\zeta, \delta))
$$

Using the inequality (3.5) of Lemma 3.2 we have

$$
\begin{aligned}
\int_{B^{\#}} \frac{|D f|^{2}}{r} d m & \lesssim \int_{B^{\#}} \frac{1}{\left|P_{\epsilon}(z)\right|} \int_{P_{\epsilon}(z)} \frac{\left(\left|D_{T} f\right|+t|D f|\right)^{2}}{r}(w) d m(w) d m(z) \\
& \lesssim \int_{(2 B)^{\#}} \frac{\left(\left|D_{T} f\right|+t|D f|\right)^{2}}{r} d m \\
& \lesssim \sigma(2 B)+t^{2} \int_{(2 B) \#} \frac{|D f|^{2}}{r} d m .
\end{aligned}
$$

Choose $t$ so that $t^{2} M<1 / 2$ and fix correponding $\delta_{0}$ (Lemma 3.2). Let $N=N(\zeta)$ be the integer such that $2^{N} B(\zeta, \delta) \subset B\left(\zeta, \delta_{0}\right) \subset 2^{N+1} B(\zeta, \delta)$. Repeating the above inequality, we have

$$
\begin{aligned}
\int_{B^{\#}} \frac{|D f|^{2}}{r} d m & \lesssim \sum_{j=1}^{N} t^{2 j-2} \sigma\left(2^{j} B\right)+t^{2 N} \int_{\left(2^{N} B\right)} \frac{|D f|^{2}}{r} d m \\
& \lesssim \sum_{j=1}^{N} t^{2 j-2} M^{j} \sigma(B)+t^{2 N} \int_{\Omega} \frac{|D f|^{2}}{r} d m \\
& \lesssim \sigma(B)+t^{2 N} \int_{\Omega} \frac{|D f|^{2}}{r} d m
\end{aligned}
$$

By (3.5) with $t$ small enough and the Carleson measure property of $r^{-1}\left|D_{T} f\right|^{2} d m$, there exist a positive constant $C$ and a compact subset $K$ of $\Omega$ such that

$$
\int_{\Omega} \frac{|D f|^{2}}{r} d m \lesssim \int_{\Omega} \frac{\left|D_{T} f\right|^{2}}{r} d m+\sup _{K}|f|^{2} \leq C
$$

Thus we have

$$
\begin{aligned}
\int_{B \#} r^{-1}|D f|^{2} d m & \lesssim \sigma(B)+t^{2 N} \\
& \lesssim \sigma(B)+t^{2 N} \sigma\left(B\left(\zeta, \delta_{0}\right)\right) \\
& \lesssim \sigma(B)+t^{2 N} M^{N} \sigma(B) \\
& \lesssim \sigma(B) .
\end{aligned}
$$

This completes proof of $(3) \Rightarrow(4)$.

(4) $\Rightarrow(1)$. Suppose now that $d \mu=r\left|\nabla_{N} f\right|^{2} d m$ is a Carleson measure. We will show that $r\left|\nabla_{T} f\right|^{2} d m$ is a Carleson measure. It then follows that 
$r|\nabla f|^{2} d m$ is a Carleson measure and, by divergence theorem, $f \in H^{2}(\Omega)$. Therefore, by Lemma 3.2 of $[\mathbf{K L}]$, we can conclude that $f \in B M O A$. (The assumption of the lemma is satisfied for the finite type domain in $\mathbb{C}^{2}$ and strongly pseudoconvex domains in $\mathbb{C}^{n}$.)

Fix a ball $B=B(\zeta, \delta) \subset \partial \Omega$. Notice that $\sigma(B(\pi(z), r(z))) \approx r(z) \tau(z)^{2}$ and that if $\zeta \in B(\pi(z), r(z))$ then $z \in \mathcal{A}_{\alpha}(\zeta)$ for some $\alpha>0$. Thus by Fubini theorem, we have

$$
\int_{B^{\#}} r\left|\nabla_{T} f\right|^{2} d m \leq \int_{2 B} \int_{\mathcal{A}_{\alpha}(\zeta)} r(z)^{2}\left|\nabla_{T} f(z)\right|^{2} \frac{d m(z)}{r(z)^{2} \tau(z)^{2}} d \sigma(\zeta) .
$$

In order to control the tangential derivative of $f$ in (4.1) in terms of the normal derivative, we need the following lemma.

Lemma 4.1. Put $K=\left\{z \in \Omega: \operatorname{dist}(z, \partial \Omega) \geq \delta_{0} / 2\right\}$. For each $\alpha>0$ there exists $\beta>0$ and a constant $C>0$ such that for all $f$ holomorphic in $\Omega$ and for all $\zeta \in \partial \Omega$,

$$
\int_{\mathcal{A}_{\alpha}(\zeta)} r^{2}\left|\nabla_{T} f\right|^{2} \frac{d m}{r^{2} \tau^{2}} \leq C \int_{\mathcal{A}_{\beta}(\zeta)} r^{3}|\nabla f|^{2} \frac{d m}{r^{2} \tau^{2}}+C \sup _{K}|f|^{2}
$$

We assume Lemma 4.1 temporarily and complete the proof of Theorem 1.1. Assume that $\delta_{0}=1$ for convenience. Since $d \mu(z)=r(z)\left|\nabla_{N} f(z)\right|^{2} d m(z)$ is a Carleson measure, we have

$$
\begin{aligned}
\int_{\mathcal{A}_{\beta}(\zeta)} r^{3}\left|\nabla_{N} f\right|^{2} \frac{d m}{r^{2} \tau^{2}} & \lesssim \sum_{j=1}^{\infty} \int_{B^{\#}\left(\zeta, 2^{-j}\right) \backslash B^{\#}\left(\zeta, 2^{-j-1}\right)} \frac{2^{-j} r\left|\nabla_{N} f\right|^{2}}{\sigma\left(B\left(\zeta, 2^{-j-1}\right)\right)} d m \\
& \lesssim \sum_{j=1}^{\infty} \frac{\sigma\left(B\left(\zeta, 2^{-j}\right)\right)}{2^{j} \sigma\left(B\left(\zeta, 2^{-j-1}\right)\right)} \\
& \leq C .
\end{aligned}
$$

Thus it follows from (4.1) and Lemma 4.1 that

$$
\int_{B^{\#}} r\left|\nabla_{T} f\right|^{2} d m \leq C \sigma(B) .
$$

This completes the proof of Theorem 1.1.

Proof of Lemma 4.1. Let us assume $\delta_{0}=1$ for convenience. Put $d V=$ $(r \tau)^{-2} d m$. For given $\alpha>0$, choose $\epsilon>0$ and $\beta>\alpha$ so that for all $z \in \mathcal{A}_{\alpha}(\zeta)$, $P_{\epsilon}(z) \subset \mathcal{A}_{\beta}(\zeta)$ (Lemma 2.2). For $\alpha>0$ and $t>0$, let

$$
S_{\alpha, t}=S_{\alpha, t}(\zeta)=\left\{z \in \mathcal{A}_{\alpha}(\zeta): t / 2<r(z)<2 t\right\} .
$$


Then, $\left|S_{\alpha, t}(\zeta)\right| \approx t^{2} \tau(\zeta, t)^{2}$. Set

$$
J_{t}=\int_{S_{\beta, t}}|\nabla f|^{2} d V
$$

Then by (3.2), for all $z \in \mathcal{A}_{\alpha}(\zeta)$ we have

$$
\begin{aligned}
\left|\nabla \nabla_{T} f(z)\right| & \lesssim\left|\nabla_{T} \nabla f(z)\right|+|\nabla f(z)| \\
& \lesssim \tau(z)^{-1} \int_{P_{\epsilon}(z)}|\nabla f| d V \\
& \lesssim \tau(z)^{-1}\left(\int_{P_{\epsilon}(z)}|\nabla f|^{2} d V\right)^{1 / 2} \\
& \lesssim \tau(z)^{-1} J_{r(z)}^{1 / 2} .
\end{aligned}
$$

Put $\pi(z)=\eta$. Then

$$
\begin{aligned}
\left|\nabla_{T} f(z)\right| & \leq \int_{r(z)}^{1}\left|\nabla \nabla_{T} f\left(\eta-t \nu_{\eta}\right)\right| d t+C \sup _{K}|f| \\
& \lesssim \int_{r(z)}^{1} \tau(\eta, t)^{-1} J_{t}^{1 / 2} d t+\sup _{K}|f| .
\end{aligned}
$$

Thus,

$$
\begin{aligned}
& \int_{\mathcal{A}_{\alpha}(\zeta)} r^{2}\left|\nabla_{T} f\right|^{2} d V \\
& \lesssim \int_{\mathcal{A}_{\alpha}(\zeta)} r^{2}\left(\int_{r}^{1} \tau(\pi(z), t)^{-1} J_{t}^{1 / 2} d t\right)^{2} d V+\sup _{K}|f|^{2} \\
& \lesssim \sum_{j=1}^{\infty} \int_{S_{\alpha, 2^{-j}}} 2^{-2 j}\left(\int_{2^{-j-1}}^{1} \tau(\pi(z), t)^{-1} J_{t}^{1 / 2} d t\right)^{2} d V+\sup _{K}|f|^{2} \\
& \lesssim \sum_{j=1}^{\infty} 2^{-2 j}\left(\int_{2^{-j-1}}^{1} \tau(\pi(z), t)^{-1} J_{t}^{1 / 2} d t\right)^{2}+\sup _{K}|f|^{2} \\
& \lesssim \int_{0}^{1} r\left(\int_{r}^{1} \tau(\pi(z), t)^{-1} J_{t}^{1 / 2} d t\right)^{2} d r+\sup _{K}|f|^{2} .
\end{aligned}
$$

Thus by Hardy's inequality we have

$$
\int_{\mathcal{A}_{\alpha}(\zeta)} r^{2}\left|\nabla_{T} f\right|^{2} d V \lesssim \int_{0}^{1} t^{3} \tau(\pi(z), t)^{-2} J_{t} d t+\sup _{K}|f|^{2} .
$$

Since $t \lesssim \tau(\pi(z), t)^{2}$ and $t \approx r(z)$ on $S_{\beta, t}$,

$$
\int_{\mathcal{A}_{\alpha}(\zeta)} r^{2}\left|\nabla_{T} f\right|^{2} d V \lesssim \int_{0}^{1} t^{2} J_{t} d t+\sup _{K}|f|^{2}
$$




$$
\lesssim \int_{\mathcal{A}_{\beta}(\zeta)} r^{3}|\nabla f|^{2} d V+\sup _{K}|f|^{2}
$$

This completes the proof.

\section{BMOA on strongly pseudo-convex domains.}

In this section, we give Carleson measure characterizations of BMOA on strongly pseudo-convex domains in $\mathbb{C}^{n}$. The proof is simply a repeat of the proof in the previous section, so we omit the proof.

Let $\Omega=\left\{z \in \mathbb{C}^{n}: r(z)>0\right\}$ be a bounded strongly pseudo-convex domain in $\mathbb{C}^{n}$ where $r$ is a smooth real valued fuction such that $|\nabla r|=1$ on $\partial \Omega$. Then, one can define a pseudo-distance (Koranyi distance) on $\partial \Omega$ by

$$
d(\zeta, \xi)=\left|\sum_{j=1}^{n} \frac{\partial r}{\partial z_{j}}(\zeta)\left(\zeta_{j}-\xi_{j}\right)\right|+|\zeta-\xi|^{2} .
$$

Balls on $\partial \Omega$ can be defined correspondingly. Then, the radius $\tau(\zeta, \delta)$ in the complex tangential direction of the ball $B(\zeta, \delta)$ becomes approximately $\delta^{1 / 2}$.

Theorem 5.1. Let $\Omega=\left\{z \in \mathbb{C}^{n}: r(z)>0\right\}$ be a bounded strongly pseudo-convex domain in $\mathbb{C}^{n}$ where $r$ is a smooth real valued fuction such that $|\nabla r|=1$ on $\partial \Omega$. Then for $f$ holomorphic in $\Omega$ the following are equivalent;

(1) $f \in B M O A$.

(2) $\quad d \mu(z)=\left(r(z)\left|\nabla_{N} f(z)\right|^{2}+\left|\nabla_{T} f(z)\right|^{2}\right) d m(z)$ is a Carleson measure.

(3) $d \mu(z)=\left|\nabla_{T} f(z)\right|^{2} d m(z)$ is a Carleson measure.

(4) $d \mu(z)=r(z)\left|\nabla_{N} f(z)\right|^{2} d m(z)$ is a Carleson measure.

\section{References}

[BDN] A. Boggess, R. Dwilewicz and A. Nagel, The hull of holomorhpy of a nonisotropic ball in a real hypersurface of finite type, Trans. of Amer. Math. Soc., 323 (1991), 209-232.

[C] D. Catlin, Estimates of invariant metrics on pseudoconvex domains of dimension two, Math. Z., 200 (1989), 429-466.

[CC] J. Choa and B. Choe, A Littlewood-Paley type identity and a characterization of BMOA, Complex Variables, 17 (1991), 15-23.

[CRW] R. Coifman, R. Rochberg and G. Weiss, Factorization theorems for Hardy spaces in several variables, Ann. Math., 103 (1976), 611-635.

[CW] R. Coifman and G. Weiss, Analyse harmonique non-commutative sur certains espaces homogenes, Lecture Notes in Math., 242, Springer-Verlag, 1971. 
[FS] C. Fefferman and E. Stein, $H^{p}$ spaces of several variables, Acta Math., 129 (1972), 137-193.

[G] S. Grellier, Behavior of holomorphic functions in complex tangential directions in a domain of finite type in $C^{n}$, Publications Matematiques, 36 (1992), 251-292.

[J] M. Jevtic, A note on the Carleson measure characterization of BMOA functions on the unit ball, Complex Variables, 17 (1992), 189-194.

[K] H. Koo, Boundary behavior of holomorphic functions on domains of finite type, Thesis, University of Wisconsin-Madison, 1993.

[KL] S. Krantz and S. Li, A note on Hardy spaces and functions of bounded mean oscillation on domains in $\mathbb{C}^{n}$, Mich. Math. J., 41 (1994), 51-71.

[Mc] J. McNeal, Boundary behavior of the Bergman kernel funciton in $\mathbb{C}^{2}$, Duke Math. J., 58(2) (1989), 499-512.

[NRSW] A. Nagel, J.-P. Rosay, E. Stein and S. Wainger, Estimates for the Bergman and Szegö kernel in $\mathbb{C}^{n}$, Ann. Math., 129 (1989), 113-149.

[NSW1] A. Nagel, E. Stein and S. Wainger, Boundary behavior of functions holomorphic in domains of finite type, Proc. Nat. Acad. Sci. USA, 78 (1981), 6596-6599.

[NSW2] , Balls and metrics defined by vector fields I: Basic properties, Acta Math., 155 (1985), 103-147.

[S2] E. Stein, Harmonic analysis, Princeton University Press, Princeton, NJ, 1993.

Received June 25, 1995 and revised March 4, 1996. The authors were supported in part by KOSEF 941-0100-015-2, GARC-KOSEF and BSRI 1995.

Korea UNIVERSITY

SEOUL 136-075, KoreA

E-mail address: kang@semi.korea.ac.kr

AND

HANKUK UNIVERSiTy OF Foreign StUdies

KYUNGKI-DO 449-850, KOREA

E-mail address: koohw@maincc.hufs.ac.kr

Note: The Reference [CRW] AbOve was typeset as [CRC] in the PRInted VERSION. 